TITLE:

\title{
Magneto-optical properties of transparent divalent iron phosphate glasses
}

$\operatorname{AUTHOR}(\mathrm{S})$ :

Akamatsu, Hirofumi; Fujita, Koji; Murai, Shunsuke; Tanaka, Katsuhisa

\section{CITATION:}

Akamatsu, Hirofumi ... [et al]. Magneto-optical properties of transparent divalent iron phosphate glasses. APPLIED PHYSICS LETTERS 2008, 92(25): 251908.

\section{ISSUE DATE:}

2008-06-23

URL:

http://hdl.handle.net/2433/84595

\section{RIGHT:}

Copyright 2008 American Institute of Physics. This article may be downloaded for personal use only. Any other use requires prior permission of the author and the American Institute of Physics. 


\title{
Magneto-optical properties of transparent divalent iron phosphate glasses
}

\author{
Hirofumi Akamatsu, Koji Fujita, Shunsuke Murai, and Katsuhisa Tanaka ${ }^{\text {a) }}$ \\ Department of Material Chemistry, Graduate School of Engineering, Kyoto University, \\ Nishikyo-ku, Kyoto 615-8510, Japan
}

(Received 10 April 2008; accepted 5 June 2008; published online 24 June 2008)

\begin{abstract}
We have prepared glasses having $x \mathrm{FeO} \cdot(100-x) \mathrm{P}_{2} \mathrm{O}_{5}(\mathrm{~mol} \%)(x=50.0,54.0,57.1)$ compositions by melting under mild reducing condition and found that these glasses exhibit fairly high transmittance in the visible range and large Faraday effect at the wavelength of about $400 \mathrm{~nm} .{ }^{57} \mathrm{Fe}$ Mössbauer spectra confirm that almost all the iron ions are present as $\mathrm{Fe}^{2+}$ in the glasses. A spin glass transition is observed at low temperatures in the temperature dependence of magnetic susceptibility. Intense optical absorption in the ultraviolet and infrared wavelength ranges occurs by the charge transfer transition from $\mathrm{O}^{2-}$ to $\mathrm{Fe}^{2+}$ and the intra-atomic $d-d$ transition, respectively. The analysis on the wavelength dependence of the Faraday rotation angle using the Van Vleck-Hebb theory has revealed that the charge transfer transition contributes more significantly to the Faraday effect owing to the large effective transition probability, which is comparable to those reported for glasses containing $4 f$ rare-earth ions. The magneto-optical figure of merit shows a maximum at around $380 \mathrm{~nm}$. (C) 2008 American Institute of Physics. [DOI: 10.1063/1.2952460]
\end{abstract}

In magnetic oxide glasses, the magnetic moments belonging to the cations such as $3 d$ transition metal and $4 f$ rare-earth ions are randomly distributed in the host glass structure. Oxide glass often shows peculiar and intriguing magnetic transition like that of a spin glass, in which magnetic moments are frozen in a random fashion, at low temperatures. The mechanism of magnetic transition observed in oxide glasses has been experimentally investigated mainly based on the temperature dependence of dc and ac magnetic susceptibilities and ${ }^{57} \mathrm{Fe}$ Mössbauer spectrum. ${ }^{1-3}$ We have revealed that $\mathrm{Fe}_{2} \mathrm{O}_{3}-\mathrm{TeO}_{2}$ and $\mathrm{Bi}_{2} \mathrm{O}_{3}-\mathrm{Fe}_{2} \mathrm{O}_{3}-\mathrm{B}_{2} \mathrm{O}_{3}$ glasses exhibit curious phenomena relevant to the spin dynamics including magnetic aging and memory effects as well as critical slowing down, indicating that the low-temperature magnetically ordered phase of these oxide glasses can be definitely regarded as a spin glass. ${ }^{4,5}$

Another interesting phenomenon associated with the magnetic oxide glasses lies in magneto-optical properties such as the Faraday effect. A great deal of attention has been paid to the Faraday effect of glasses containing $4 f$ rare-earth ions such as $\mathrm{Eu}^{2+}$ and $\mathrm{Tb}^{3+} \cdot{ }^{6-10}$ The glasses with high concentration of such rare-earth ions show rather high transmittance and large Faraday effect in the visible to ultraviolet wavelength region. These glasses can be applied as an optical isolator, an optical switch, and an optical modulator working in such a short wavelength range. On the other hand, there exist few reports on the Faraday effect of glasses containing $3 d$ transition metal ions because the glasses bearing only a few molar percent of these ions often show intense absorption in the visible range. Nevertheless, in this study, we have prepared phosphate glasses containing a large amount of $\mathrm{Fe}^{2+}$ ions, which are highly transparent in the wavelength range from 400 to $700 \mathrm{~nm}$; we have examined their magnetic susceptibilities, optical absorption spectra, and Faraday rotation angles. Some iron phosphate glasses melted at various temperatures and under reducing conditions have been characterized so far, ${ }^{11-13}$ but there are no reports on iron phosphate glasses containing only $\mathrm{Fe}^{2+}$ ions,

${ }^{a)}$ Electronic mail: tanaka@dipole7.kuic.kyoto-u.ac.jp. the concentration of which is as high as several ten percent or so. Besides, although the reduction of $\mathrm{Fe}^{3+}$ into $\mathrm{Fe}^{2+}$ ions was completely achieved for an iron aluminosilicate glass system, the optical absorption and Faraday effect were not measured. ${ }^{3}$ We demonstrate that, in addition to the high transmittance in the visible range, the present phosphate glasses containing divalent iron ions exhibit large Faraday effect in the near-ultraviolet to blue range, which meets the emission from the blue laser diodes. The dependence of the Faraday rotation angle on wavelength can be analyzed in terms of the Van Vleck-Hebb theory. This analysis leads to the effective transition probability comparable to those for the glasses containing rare-earth ions.

Glasses having $x \mathrm{FeO} \cdot(100-x) \mathrm{P}_{2} \mathrm{O}_{5}(\mathrm{~mol} \%)(x=50.0$, 54.0,57.1) compositions were prepared by a conventional melt quenching method using reagent grade $\mathrm{Fe}_{2} \mathrm{O}_{3}$ and $\mathrm{H}_{3} \mathrm{PO}_{4}$ aqueous solution as starting materials. $\mathrm{Fe}_{2} \mathrm{O}_{3}$ powder and $\mathrm{H}_{3} \mathrm{PO}_{4}$ aqueous solution were mixed and the mixture was heated at $180^{\circ} \mathrm{C}$ to eliminate water. The mixture was then melted in an alumina crucible at $1200{ }^{\circ} \mathrm{C}$ for $30 \mathrm{~min}$ in air. The melt was poured onto a stainless steel plate to obtain glass, which showed black color due to the presence of $\mathrm{Fe}^{3+}$ ions. The resultant glass was pulverized, and the glass powder was remelted in a glassy carbon crucible placed in an alumina crucible with an alumina lid at $1200{ }^{\circ} \mathrm{C}$ for $30 \mathrm{~min}$ in air. The melt was quenched by being pressed with two stainless steel plates. Surprisingly, the glasses thus obtained were fairly transparent to the naked eye. The as-quenched glasses were annealed at $460-480{ }^{\circ} \mathrm{C}$ for $3 \mathrm{~h}$ in an atmosphere of $\mathrm{Ar}(95 \mathrm{vol} \%) / \mathrm{H}_{2}(5 \mathrm{vol} \%)$ to prevent the glasses from oxidizing. X-ray diffraction analysis with $\mathrm{Cu} \mathrm{K} \alpha$ radiation confirmed the absence of crystalline phases in the glasses. Although phosphate glasses sometimes show relatively poor chemical durability, changes in appearance such as devitrification have not been observed for the present phosphate glasses even after they were set in air for more than 4 months. The glass samples were polished so that their thickness was $0.4-0.5 \mathrm{~mm}$ and subjected to Faraday effect and optical absorption measurements. The Faraday effect measurements were performed at room temperature by using 


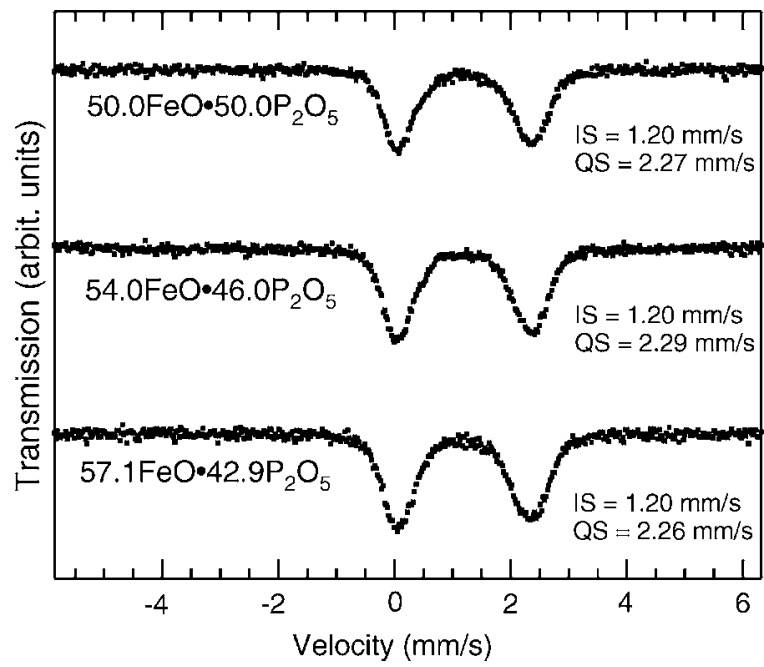

FIG. $1 .{ }^{57} \mathrm{Fe}$ Mössbauer spectra at room temperature for the $\mathrm{FeO}-\mathrm{P}_{2} \mathrm{O}_{5}$ glasses with 50.0, 54.0, and $57.1 \mathrm{~mol} \%$ of $\mathrm{FeO}$. IS and QS denote the isomer shift and the quadrupole splitting, respectively, for the doublet assigned to $\mathrm{Fe}^{2+}$.

a commercial measurement system for Faraday and Kerr effects (JASCO, model K-250). The wavelength was varied from 350 to $850 \mathrm{~nm}$ and the applied magnetic field was fixed to be $15 \mathrm{kOe}$. The optical absorption spectra were recorded using a spectrophotometer (JASCO, V-570). The transparent glasses were pulverized and subjected to ${ }^{57} \mathrm{Fe}$ Mössbauer effect and magnetic susceptibility measurements. The ${ }^{57} \mathrm{Fe}$ Mössbauer measurements were performed at room temperature by using ${ }^{57} \mathrm{Co}$ in metallic $\mathrm{Rh}$ as a $\gamma$-ray source. The magnetic susceptibility under zero-field cooling (ZFC) and field cooling (FC) conditions was measured in the temperature range from 2 to $300 \mathrm{~K}$ with a dc magnetic field of 50 Oe applied by utilizing a superconducting quantum interference device magnetometer (Quantum Design, model MPMS-XL).

The ${ }^{57} \mathrm{Fe}$ Mössbauer spectra of the glasses are depicted in Fig. 1. All the spectra are composed of a paramagnetic doublet attributable to $\mathrm{Fe}^{2+}$ ions; absorption peaks ascribed to $\mathrm{Fe}^{3+}$ ions are barely observed in all the spectra. The Mössbauer spectra of glasses prepared by melting only in air indicate that more than $80 \%$ of iron ions are present as $\mathrm{Fe}^{3+}$ in the glasses, although neither the spectra nor the Mössbauer parameters derived from the spectra are shown here. The remelting in a glassy carbon crucible could reduce almost all $\mathrm{Fe}^{3+}$ ions into $\mathrm{Fe}^{2+}$ ions. It is much easier to obtain the $\mathrm{Fe}^{2+}$ ion with this technique compared with the conventional method such as melting under a flow of reducing gas. The isomer shift (IS) and quadrupole splitting for the glasses after the remelting are summarized in Fig. 1. The value of IS $=1.20 \mathrm{~mm} / \mathrm{s}$ corresponds to an $\mathrm{Fe}^{2+}$ octahedrally coordinated by $\mathrm{O}^{2-}$ ions.

Figure 2 shows the optical absorption spectra of the glasses, which exhibit fairly high transmittance in the wavelength range from 400 to $700 \mathrm{~nm}$. The intense optical absorptions in the ultraviolet and infrared regions are ascribable to the charge transfer transition from $\mathrm{O}^{2-}$ to $\mathrm{Fe}^{2+}$ and intra-atomic $d$ - $d$ crystal field transition, respectively. ${ }^{14} \mathrm{Al}-$ though the presence of $\mathrm{Fe}^{3+}$ ions cannot be detected in the Mössbauer spectra, very weak absorption bands attributed to a tiny amount of $\mathrm{Fe}^{3+}$ ions are observed at $400-550 \mathrm{~nm} .{ }^{15}$ As the value of $x$ is increased, the amount of $\mathrm{Fe}^{3+}$ ion is apt Downloaded 29 Jun 2009 to 130.54.110.22. Redistribution subjec

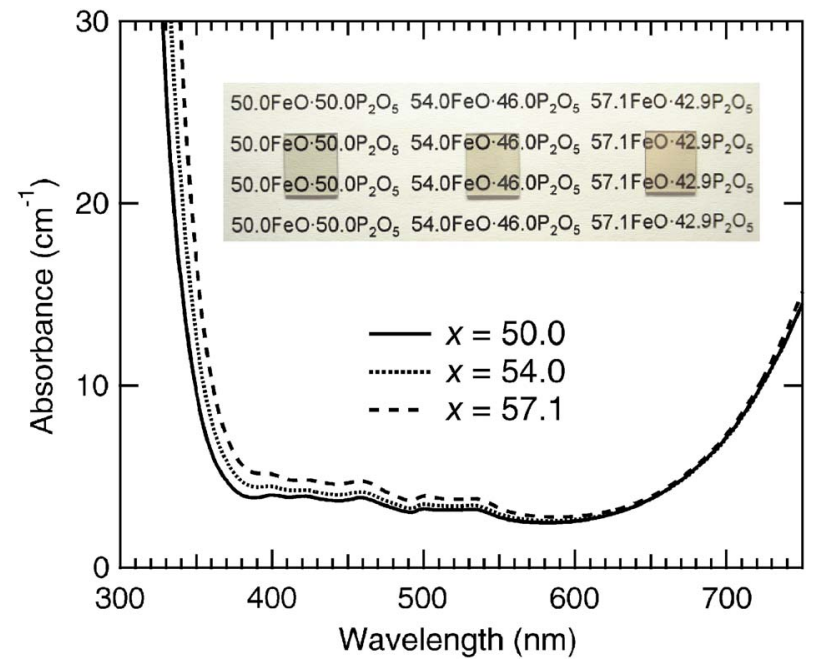

FIG. 2. (Color online) Optical absorption spectra for the $x \mathrm{FeO} \cdot(100$ $-x) \mathrm{P}_{2} \mathrm{O}_{5}(\mathrm{~mol} \%)$ glasses with $x=50.0,54.0$, and 57.1. The inset depicts pictures of the glasses with the thickness of about $450 \mu \mathrm{m}$.

to increase and hence the glass turns its color from blue greenish into orangish (see the inset of Fig. 2).

Figure 3 illustrates the temperature dependence of magnetic susceptibility, $\chi(T)$, and its reciprocal, $\chi^{-1}(T)$, for the $57.1 \mathrm{FeO} \cdot 42.9 \mathrm{P}_{2} \mathrm{O}_{5}$ glass. The linear dependence of $\chi^{-1}(T)$ on $T$ at high temperatures $(30 \mathrm{~K} \leqslant T \leqslant 300 \mathrm{~K})$ reveals that these glasses are paramagnetic in the temperature region; the linear part is describable in terms of the following CurieWeiss law:

$$
\chi^{-1}(T)=\frac{3 k_{B}\left(T-\theta_{W}\right)}{N M_{B}^{2} \mu_{B}^{2}},
$$

where $\theta_{W}$ is the Weiss temperature, $N$ is the number of magnetic ions per unit mass, $\mu_{B}$ is the Bohr magneton, $M_{B}$ is the effective number of Bohr magnetons, and $k_{B}$ is the Boltzmann constant. The value of $M_{B}=5.21$ obtained by fitting Eq. (1) to the experimental data of $\chi^{-1}(T)$ at high temperatures is slightly higher than the theoretical spin-only value of $\mathrm{Fe}^{2+}$, i.e., 4.82, presumably due to the contribution of unquenched orbital magnetic moment. The inset of Fig. 3 shows $\chi(T)$ in the low-emperature region. $\chi(T)$ in ZFC and FC deviate from each other at the temperature where $\chi(T)$ in the ZFC run exhibits a maximum. This behavior of $\chi(T)$ is characteristic of the spin glass. The spin freezing tempera-

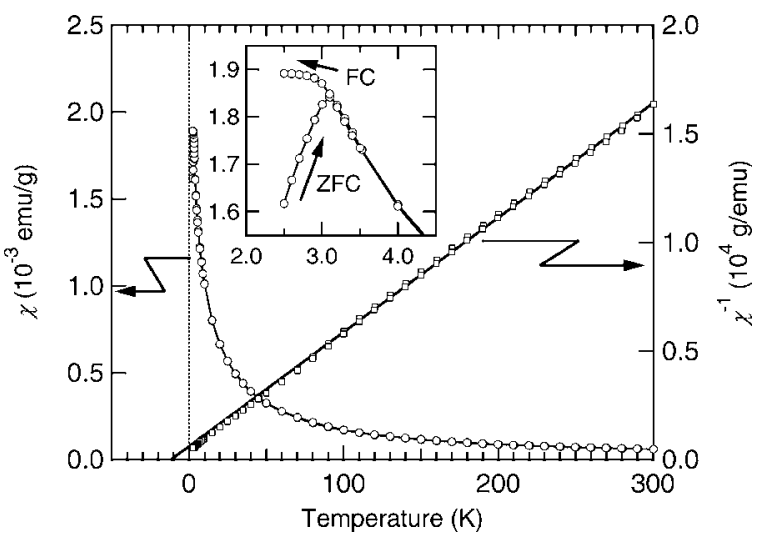

FIG. 3. Temperature dependence of dc magnetic susceptibility and its reciprocal for the $57.1 \mathrm{FeO} \cdot 42.9 \mathrm{P}_{2} \mathrm{O}_{5}$ glass. The inset illustrates the enlarged

view of the low-temperature region. 


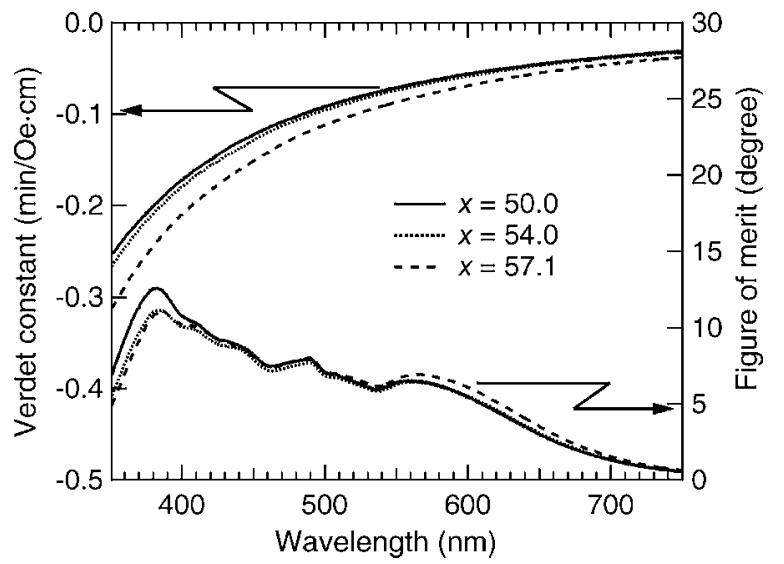

FIG. 4. Wavelength dependence of the Verdet constant and magneto-optical figure of merit for the $x \mathrm{FeO} \cdot(100-x) \mathrm{P}_{2} \mathrm{O}_{5}(\mathrm{~mol} \%)$ glasses with $x=50.0$, 54.0, and 57.1.

tures are, 2.2, 2.6, and $3.1 \mathrm{~K}$ and $\theta_{W}$ 's are $-8.6,-9.0$, and $-11.6 \mathrm{~K}$ for $x \mathrm{FeO} \cdot(100-x) \mathrm{P}_{2} \mathrm{O}_{5}$ glasses with $x=50.0,54.0$, and 57.1, respectively. The negative value of $\theta_{W}$ means that the antiferromagnetic interaction is dominant among the magnetic moments of $\mathrm{Fe}^{2+}$ ions in the glasses.

The wavelength dependence of the Verdet constant $V$ for the glasses is illustrated in Fig. 4. For paramagnets, $V$ $=\theta /(H l)$, where $\theta$ is the Faraday rotation angle, $H$ is the applied magnetic field, and $l$ is the length of light path in the material. The absolute value of $V$ monotonically increases as the wavelength of incident light, $\lambda$, becomes shorter. A similar tendency was observed for the rare-earth-containing glasses. The relation between $V$ and $\lambda$ for the glasses containing rare-earth ions has been analyzed in terms of the Van Vleck-Hebb theory using the following equation:

$$
V=\frac{4 \pi^{2} \chi}{g \mu_{B} c h} \sum_{i} C_{i}\left(1-\frac{\lambda^{2}}{\lambda_{i}^{2}}\right)^{-1},
$$

where $g$ is the Landé factor, $c$ is the velocity of light, $h$ is the Planck constant, $C_{i}$ is related to the transition probability, and $\lambda_{i}$ is the transition wavelength. The subscript $i$ denotes the electronic transition which contributes to the Faraday effect. The approximation taking only single electronic transition into account has been used to explain the wavelength dependence of $V$ of rare-earth-containing glasses. ${ }^{6-9}$ However, the dependence of $V$ of the iron phosphate glasses on $\lambda$ cannot be interpreted by this single oscillator model but described well by considering two optical transitions, i.e., the charge transfer transition and the crystal field transition in the ultraviolet and infrared regions, respectively. For instance, the analysis using Eq. (2) yields $C_{1}=8.6$ $\times 10^{-45} \mathrm{~J} \mathrm{~cm}^{3}, \lambda_{1}=214 \mathrm{~nm}, C_{2}=1.1 \times 10^{-46} \mathrm{~J} \mathrm{~cm}^{3}$, and $\lambda_{2}$ $=1100 \mathrm{~nm}$ for $57.1 \mathrm{FeO} \cdot 42.9 \mathrm{P}_{2} \mathrm{O}_{5}$ glass. The value of $C_{1}$ corresponding to the charge transfer transition is much larger than that of $C_{2}$ corresponding to the $d-d$ transition. This fact indicates that the charge transfer transition mainly contributes to the Faraday effect due to the large oscillator strength.

Also shown in Fig. 4 is the magneto-optical figure of merit defined as $|\theta| / \alpha$, where $\theta$ is the Faraday rotation angle and $\alpha$ is the absorbance. $|\theta| / \alpha$ is an important factor from the point of view of practical applications. For all the glasses, $|\theta| / \alpha$ exhibits a maximum at around $380 \mathrm{~nm}$. For instance, $|\theta| / \alpha$ of $50.0 \mathrm{FeO} \cdot 50.0 \mathrm{P}_{2} \mathrm{O}_{5}$ glass is $13^{\circ}$ at $380 \mathrm{~nm}$ under $H=15 \mathrm{kOe}$. Since the wavelength such as $380 \mathrm{~nm}$ corre- sponds to the emission of blue laser diodes, the present glasses are expected as a candidate for optical devices such as an optical isolator in such a short wavelength region. The amplitude of $V$ for the present glasses is several times smaller than or comparable to those for the rare-earth-containing glasses; for instance, at $405 \mathrm{~nm}, V$ is -0.204 and $-0.560 \mathrm{~min} / \mathrm{Oe} \mathrm{cm}$ for $57.1 \mathrm{FeO} \cdot 42.9 \mathrm{P}_{2} \mathrm{O}_{5}$ and $25.4 \mathrm{~Tb}_{2} \mathrm{O}_{3} \cdot 74.6 \mathrm{P}_{2} \mathrm{O}_{5}$ glasses, respectively, both of which are phosphate glasses and have almost the same effective transition wavelength. ${ }^{16}$ Another advantage of the present glasses lies in the fact that both iron oxide and phosphoric acid, i.e., the starting materials for the glasses, are much more inexpensive and available when compared with the rare-earth oxides which have been practically utilized as a component of glass materials for Faraday rotators.

In conclusion, the glasses with $x \mathrm{FeO} \cdot(100-x) \mathrm{P}_{2} \mathrm{O}_{5}$ $(\mathrm{mol} \%)(x=50.0,54.0,57.1)$ compositions exhibit farely high transmittance in the visible region, although the glasses contain a large amount of $3 d$ transition metal ions. The glasses have large Faraday rotation angle in the near-ultraviolet to blue region. The large effective transition probability comparable to those reported for the rare-earth-containing glasses stems from the large oscillator strength of charge transfer transition from $\mathrm{O}^{2-}$ to $\mathrm{Fe}^{2+}$. Considering that $\mathrm{Fe}$ is an abundant element in the crust of the earth, the achievement of FeO-based glass materials which shows high transmittance as well as large Faraday effect in the near-ultraviolet to blue region is of great importance.

The authors would like to thank Dr. M. Tosaki of Radioisotope Research Center, Kyoto University for the Mössbauer effect measurements. This research was partially supported by the Ministry of Education, Science, Sports and Culture, Grant-in-Aid for Scientific Research (B), (19360298, 2007). One of the authors (H.A.) thanks the Grant-in-Aid for Fellow (No. 20-6726) from Japan Society of the Promotion Science (JSPS).

${ }^{1}$ R. A. Verhelst, R. W. Kline, A. M. de Graat, and H. O. Hooper, Phys. Rev. B 11, 4427 (1975).

${ }^{2}$ J. P. Sanchez and J. M. Friedt, J. Phys. (Paris) 43, 1707 (1982).

${ }^{3}$ J. P. Sanchez, J. M. Friedt, R. Horne, and A. J. Van Duyneveldt, J. Phys. C 17, 127 (1984).

${ }^{4}$ H. Akamatsu, K. Tanaka, K. Fujita, and S. Murai, Phys. Rev. B 74, 012411 (2006).

${ }^{5}$ H. Akamatsu, K. Tanaka, K. Fujita, and S. Murai, J. Magn. Magn. Mater. 310, 1506 (2007).

${ }^{6}$ N. F. Borrelli, J. Chem. Phys. 41, 3289 (1964).

${ }^{7}$ K. Tanaka, K. Hirao, and N. Soga, Jpn. J. Appl. Phys., Part 1 34, 4825 (1995).

${ }^{8}$ K. Tanaka, K. Fujita, N. Soga, J. Qiu, and K. Hirao, J. Appl. Phys. 83, 840 (1997).

${ }^{9}$ K. Tanaka, K. Fujita, N. Matsuoka, K. Hirao, and N. Soga, J. Mater. Res. 13, 1989 (1998).

${ }^{10}$ T. Hayakawa, M. Nogami, N. Nishi, and N. Sawanobori, Chem. Mater. 14, 3223 (2002).

${ }^{11}$ G. K. Marasinghe, M. Karabulut, C. S. Ray, D. E. Day, M. G. Shumsky, W. B. Yelon, C. H. Booth, P. G. Allen, and D. K. Shuh, J. Non-Cryst. Solids 222, 144 (1997).

${ }^{12}$ C. S. Ray, X. Fang, M. Karabulut, G. K. Marasinghe, and D. E. Day, J. Non-Cryst. Solids 249, 1 (1999).

${ }^{13}$ M. Karabulut, G. K. Marasinghe, C. S. Ray, D. E. Day, G. D. Waddill, C. H. Booth, P. G. Allen, J. J. Bucher, D. L. Caulder, and D. K. Shuh, J. Non-Cryst. Solids 306, 182 (2002).

${ }^{14}$ D. A. Nolet, J. Non-Cryst. Solids 37, 99 (1980).

${ }^{15}$ R. Carl, S. Gerlach, and C. Rüssel, J. Non-Cryst. Solids 352, 244 (2007).

${ }^{16}$ S. B. Berger, C. B. Rubinstein, C. R. Kurkjian, and A. W. Treptow, Phys. Rev. 133, A723 (1964). 